\title{
Stationary Distribution and Dynamic Behaviour of a Stochastic SIVR Epidemic Model with Imperfect Vaccine
}

\author{
Driss Kiouach (iD) and Lahcen Boulaasair \\ MSTI (Modelling, Systems and Technologies of Information) Team, High School of Technology, Ibn Zohr University, Agadir, Morocco \\ Correspondence should be addressed to Driss Kiouach; d.kiouach@uiz.ac.ma
}

Received 19 April 2018; Accepted 2 July 2018; Published 18 July 2018

Academic Editor: Oluwole D. Makinde

Copyright (c) 2018 Driss Kiouach and Lahcen Boulaasair. This is an open access article distributed under the Creative Commons Attribution License, which permits unrestricted use, distribution, and reproduction in any medium, provided the original work is properly cited.

\begin{abstract}
We consider a stochastic SIVR (susceptible-infected-vaccinated-recovered) epidemic model with imperfect vaccine. First, we obtain critical condition under which the disease is persistent in the mean. Second, we establish sufficient conditions for the existence of an ergodic stationary distribution to the model. Third, we study the extinction of the disease. Finally, numerical simulations are given to support the analytical results.
\end{abstract}

\section{Introduction}

Mathematical models have been an unavoidable tool in analyzing the mechanisms of infectious diseases. Most modelers were inspired by the works in [1-3]. For controlling the spread of diseases, vaccination is considered the most effective way to reduce both the morbidity and mortality of individuals (see [4-6]). In this paper, we consider the deterministic SIVR (susceptible-infected-vaccinated-recovered) model:

$$
\begin{aligned}
d S & =[\mu-(\mu+\phi) S-\beta S I] d t, \\
d I & =[\beta(S+\eta V) I-(\mu+\gamma) I] d t, \\
d V & =[\phi S-\eta \beta V I-\mu V] d t, \\
d R & =[\gamma I-\mu R] d t,
\end{aligned}
$$

where S, I, and R denote the densities of susceptible, infected, and recovered individuals, respectively. $\mathrm{V}$ denotes the density of individuals who are immune to an infection as result of vaccination. below:

The parameters involved in the system are described

$\beta$ : the average number of contacts per infected per unit time

$\mu$ : the recruitment rate and the death rate $\gamma$ : recovery rate of infected individuals

$\phi$ : the rate at which susceptible individuals are moved into the vaccination process

$\eta$ : a positive factor satisfying $0 \leq \eta \leq 1$, and $\eta=0$ means that the vaccine is perfectly effective and $\eta=1$ means that the vaccine has no effect

In model (1), the fundamental parameter that governs the spread of the disease into a population is the basic reproduction number denoted by $R_{0}$. It can be thought of as the number of cases one case generates on average over the course of its infectious period in an otherwise uninfected population (see [7]).

Let us denote by $R_{0}=\beta /(\mu+\gamma)$ the basic reproduction number of model (1) and by $R_{\phi}=R_{0}((\mu+\eta \phi) /(\mu+\phi))$ the basic reproduction number in a population in which a proportion $\phi$ had been vaccinated. It is known that, in the absence of the disease, there is a unique disease-free equilibrium $\left(S_{0}, I_{0}, V_{0}, R_{0}\right)=(\mu /(\mu+\phi), 0, \phi /(\mu+\phi), 0)$ which is globally asymptotically stable if $R_{\phi}<1$. If $R_{\phi}>1$ for some parameters values, the model exhibits a backward bifurcation leading to the existence of multiple endemic equilibria and news subthreshold, which may be important when it comes to designing vaccination strategies (see $[8,9]$ ).

It is well known that epidemic models are inevitably affected by the environmental noise that influences the 
dynamic behaviour of the epidemic models (see [10-14]). For this, inspired by the works in [15-17], Tornatore et al. [18] have formulated and studied a stochastic version of model (1) by replacing the contact rate $\beta$ in system (1) by $\beta+\sigma(d W(t) / d t)$, where $W(t)$ denotes the standard Brownian motion and $\sigma^{2}$ denotes the intensity of the white noise. Then, system (1) becomes as follows:

$$
\begin{aligned}
d S= & {[\mu-(\mu+\phi) S-\beta S I] d t-\sigma S I d W(t), } \\
d I= & {[\beta(S+\eta V) I-(\mu+\gamma) I] d t } \\
& +\sigma(S+\eta V) I d W(t), \\
d V= & {[\phi S-\eta \beta V I-\mu V] d t-\sigma \eta V I d W(t), } \\
d R= & {[\gamma I-\mu R] d t . }
\end{aligned}
$$

Dianli Zhao and Sanling Yuan [19] have obtained conditions ensuring the persistence and extinction of model (2) and have found a threshold $R_{\phi}^{S}=(1 /(\mu+\gamma))[\beta((\mu+\eta \phi) /(\mu+$ $\left.\phi))-\left(\sigma^{2} / 2\right)((\mu+\eta \phi) /(\mu+\phi))^{2}\right]$ whose value below 1 or above 1 can determine the extinction and persistence of the epidemic under mild extra conditions.

In this paper, we assume that the multiplicative noise sources are linear in $S(t), I(t)$ and $V(t)$, according to [20]. Note that recovered population has no effect on the dynamics of $S, I$, and $V$. Then, following this approach, we obtain the following reduced stochastic SIVR model:

$$
\begin{aligned}
d S & =[\mu-(\mu+\phi) S-\beta S I] d t+\sigma_{1} S d B_{1}(t), \\
d I & =[\beta(S+\eta V) I-(\mu+\gamma) I] d t+\sigma_{2} I d B_{2}(t), \\
d V & =[\phi S-\eta \beta V I-\mu V] d t+\sigma_{3} V d B_{3}(t),
\end{aligned}
$$

where $B_{1}(t), B_{2}(t)$, and $B_{3}(t)$ are standard independent Brownian motions and $\sigma_{i}^{2}$ is a positive constant, for all $i \in\{1,2,3\}$.

This paper is organized as follows. In Section 2, we present some lemmas concerning the existence of a global positive solution and ergodic stationary distribution. In Section 3, we prove that the disease is persistent under one condition. In Section 4, we establish sufficient conditions for the existence of a unique ergodic stationary distribution. In Section 5, we determine a condition under which the disease goes to extinction. In the last section, we introduce some examples and numerical simulations to confirm our results.

\section{Preliminaries}

Throughout this paper, let $\left(\Omega, \mathscr{F},\left(\mathscr{F}_{t}\right)_{t \geq 0}, \mathbb{P}\right)$ be a complete probability space with a filtration $\left(\mathscr{F}_{t}\right)_{t \geq 0}$ satisfying the usual conditions (i.e., it is increasing and right continuous, while $\mathscr{F}_{0}$ contains all $\mathbb{P}$-null sets). Moreover, let $\mathbb{R}_{+}^{d}=$ $\left\{\left(x_{1}, \ldots, x_{d}\right) \in \mathbb{R}^{d}: x_{i}>0, i=1, \ldots, d\right\}$. If $f$ is a bounded function on $\mathbb{R}_{+}$, define $\hat{f}=\sup _{t \in[0, \infty)} f(t)$ and $\hat{f}=$ $\inf _{t \in[0, \infty)} f(t)$. We define also $\langle h\rangle=(1 / t) \int_{0}^{t} h(s) d s$.

The following theorem concerns the existence and uniqueness of the global positive solution. Since the proof is almost the same as that in [21, Lemma 2.1 and Lemma 2.2], we omit it here.

Theorem 1. For any initial value $(S(0), I(0), V(0)) \in \mathbb{R}_{+}^{3}$, there is a unique solution $(S(t), I(t), v(t)) \in \mathbb{R}_{+}^{3}$ of system (3) on $t \geq 0$ and the solution will remain in $\mathbb{R}_{+}^{3}$ with probability one. The solution $(S(t), I(t), v(t))$ has the following properties:

$$
\begin{aligned}
& \lim _{t \rightarrow \infty} \frac{S(t)}{t}=0, \\
& \lim _{t \rightarrow \infty} \frac{I(t)}{t}=0, \\
& \lim _{t \rightarrow \infty} \frac{V(t)}{t}=0,
\end{aligned}
$$

$$
\begin{gathered}
\limsup _{t \rightarrow \infty} \frac{\log S(t)}{t} \leq 0, \\
\limsup _{t \rightarrow \infty} \frac{\log I(t)}{t} \leq 0, \\
\limsup _{t \rightarrow \infty} \frac{\log V(t)}{t} \leq 0,
\end{gathered}
$$

a.s.,

Furthermore, if $\mu>\max \left(\sigma_{1}^{2}, \sigma_{2}^{2}, \sigma_{3}^{2}\right) / 2$, then

$$
\begin{aligned}
& \lim _{t \rightarrow \infty} \frac{\int_{0}^{t} S(r) d B_{1}(r)}{t}=0, \\
& \lim _{t \rightarrow \infty} \frac{\int_{0}^{t} I(r) d B_{2}(r)}{t}=0, \\
& \lim _{t \rightarrow \infty} \frac{\int_{0}^{t} V(r) d B_{3}(r)}{t}=0,
\end{aligned}
$$

a.s.

Now, we consider the $d$-dimensional stochastic differential equation:

$$
\begin{array}{r}
d x(t)=f(x(t), t) d t+g(x(t), t) d B(t) \\
\text { for all } t \geq t_{0},
\end{array}
$$

with initial value $x(0)=x_{0} \in \mathbb{R}^{d}$. $B(t)$ denotes an n-dimensional standard Brownian motion defined on the complete probability space $\left(\Omega, \mathscr{F},\left\{\mathscr{F}_{t}\right\}_{t \geq 0}, \mathbb{P}\right)$. Let us denote by $\mathscr{C}^{2,1}\left(\mathbb{R}^{d} \times\left[t_{0}, \infty\right] ; \mathbb{R}_{+}\right)$the family of all nonnegative functions $V(x, t)$ defined on $\mathbb{R}^{d} \times\left[t_{0}, \infty\right] \mathbb{R}^{d} \times\left[t_{0}, \infty\right]$ such 
that they are continuously twice differentiable in $x$ and once in $t$. The differential operator $L$ of (6) is defined by [22]

$$
\begin{aligned}
L= & \frac{\partial}{\partial t}+\sum_{i=1}^{d} f_{i}(x, t) \frac{\partial}{\partial x_{i}} \\
& +\frac{1}{2} \sum_{i, j=1}^{d}\left[g^{T}(x, t) g(x, t)\right]_{i j} \frac{\partial^{2}}{\partial x_{i} \partial x_{j}} .
\end{aligned}
$$

If $L$ acts on a function $V \in \mathscr{C}^{2,1}\left(\mathbb{R}^{d} \times\left[t_{0}, \infty\right] ; \mathbb{R}_{+}\right)$, then

$$
\begin{aligned}
L V(x, t)= & V_{t}(x, t)+V_{x}(x, t) f(x, t) \\
& +\frac{1}{2} \operatorname{trace}\left[g^{T}(x, t) V_{x x}(x, t) g(x, t)\right],
\end{aligned}
$$

where

$$
\begin{aligned}
V_{t} & =\frac{\partial V}{\partial t} \\
V_{x} & =\left(\frac{\partial V}{\partial x_{1}}, \ldots, \frac{\partial V}{\partial x_{d}}\right), \\
V_{x x} & =\left(\frac{\partial^{2} V}{\partial x_{i} \partial x_{j}}\right)_{d \times d} .
\end{aligned}
$$

By Itô's formula, we get

$$
\begin{aligned}
d V(x(t), t)= & L V(x(t), t) d t \\
& +V_{x}(x(t), t) g(x(t), t) d B(t) .
\end{aligned}
$$

Next, we shall present a lemma that gives a criterion for the existence of an ergodic stationary distribution to system (3).

Let $X(t)$ be a homogeneous Markov process in $E_{d}\left(E_{d}\right.$ denotes $d$-dimensional Euclidian space) and it is described by the following stochastic differential equation:

$$
d X(t)=h(X(t)) d t+\sum_{r=1}^{k} g_{r}(X(t)) d B_{r}(t)
$$

The diffusion matrix is defined as follows:

$$
\begin{aligned}
A(X) & =\left(a_{i j}(x)\right), \\
a_{i j}(X) & =\sum_{r=1}^{k} g_{r}^{i}(X) g_{r}^{j}(X) .
\end{aligned}
$$

Lemma 2 (see [23], Chapter 4). The Markov process $X(t)$ has a unique ergodic stationary distribution $\pi($.) if there exists a bounded domain $D \subset E_{d}$ with regular boundary $\Gamma$ and

(H.1): there is a positive number $\lambda$ such that $\sum_{i, j=1}^{d} a_{i j}(X) \xi_{i} \xi_{j} \geq \lambda|\xi|^{2}, X \in D, \xi \in \mathbb{R}^{d}$,

(H.2): there exists a nonnegative $\mathscr{C}^{2}$-function $V$ such that $L V$ is negative for any $E_{d} \backslash D$. Then

$$
\begin{aligned}
\mathbb{P}_{x} & \left\{\lim _{t \rightarrow \infty} \frac{1}{t} \int_{0}^{t} \Phi(X(s)) d s=\int_{E_{d}} \Phi(x) \pi(x) d x\right\} \\
& =1
\end{aligned}
$$

for all $x \in E_{d}$, where $\Phi($.$) is a function integrable with respect$ to the measure $\pi$.

Now, we consider the following one-dimensional homogeneous Markov process:

$$
\begin{aligned}
& d X(t)=b(X(t)) d t+\sigma(X(t)) d B(t), \\
& X\left(t_{0}\right)=X_{0}, \quad \text { for all } t \geq t_{0}
\end{aligned}
$$

where $b(X)$ and $\sigma(X)$ are measurable functions from $\mathbb{R}$ to $\mathbb{R}$ and $B(t)$ is the Brownian motion. It is assumed that the functions $b(X), \sigma(X)$, and $1 / \sigma(X)$ are locally bounded.

(H.3): the functions $b(X)$ and $\sigma(X)$ are such that

$$
\begin{aligned}
Y(x) & =\int_{0}^{x} \exp \left[-2 \int_{0}^{s} \frac{b(u)}{\sigma^{2}(u)} d u\right] d s \longrightarrow \pm \infty \\
& \text { as } x \longrightarrow \pm \infty \\
C & =\int_{-\infty}^{+\infty} \frac{1}{\sigma^{2}(x)} \exp \left[2 \int_{0}^{x} \frac{b(u)}{\sigma^{2}(u)} d u\right] d x<\infty
\end{aligned}
$$

Lemma 3 (see [24], Theorem 1.13). If (H.3) is satisfied, then the stochastic process (14) has ergodic properties with the density given by

$$
\pi(x)=\frac{1}{C \sigma^{2}(x)} \exp \left[2 \int_{0}^{x} \frac{b(u)}{\sigma^{2}(u)} d u\right] .
$$

\section{Persistence}

Definition 4. System (3) is said to be persistent in the mean, if

$$
\liminf _{t \rightarrow \infty}\langle I(t)\rangle>0 \quad \text { a.s. }
$$

Theorem 5. If $R_{0}^{S}>1$, then the disease will be persistent in mean; that is,

$$
\begin{aligned}
& \liminf _{t \rightarrow \infty} \beta(a+b+\eta c)\langle I(t)\rangle \\
& \quad \geq\left(\mu+\gamma+\frac{\sigma_{2}^{2}}{2}\right)\left(R_{0}^{S}-1\right), \quad \text { a.s. }
\end{aligned}
$$

where

$$
\begin{aligned}
a & =\frac{\beta \mu}{\left(\mu+\phi+\sigma_{1}^{2} / 2\right)^{2}}, \\
b & =\frac{\eta \beta \mu \phi}{\left(\mu+\phi+\sigma_{1}^{2} / 2\right)^{2}\left(\mu+\sigma_{3}^{2} / 2\right)}, \\
c & =\frac{\eta \beta \mu \phi}{\left(\mu+\phi+\sigma_{1}^{2} / 2\right)\left(\mu+\sigma_{3}^{2} / 2\right)^{2}}, \\
R_{0}^{S}= & \frac{\beta \mu}{\left(\mu+\phi+\sigma_{1}^{2} / 2\right)\left(\mu+\gamma+\sigma_{2}^{2} / 2\right)} \\
& +\frac{\eta \beta \mu \phi}{\left(\mu+\phi+\sigma_{1}^{2} / 2\right)\left(\mu+\gamma+\sigma_{2}^{2} / 2\right)\left(\mu+\sigma_{3}^{2} / 2\right)} .
\end{aligned}
$$


Proof. Set

$$
V_{1}=-\log I-(a+b) \log S-c \log V
$$

where positive constants $a, b$, and $c$ will be determined later. We apply Itô's formula on $V_{1}$; then we get

$$
\begin{aligned}
& d V_{1}=\left[-\frac{a \mu}{S}-\frac{b \mu}{S}+(a+b)(\mu+\phi)+\beta(a+b) I\right. \\
& -\beta S-\eta \beta V+(\mu+\gamma)-\frac{c \phi S}{V}+c \eta \beta I+\mu c+\frac{a}{2} \sigma_{1}^{2} \\
& \left.+\frac{b}{2} \sigma_{1}^{2}+\frac{\sigma_{2}^{2}}{2}+\frac{c}{2} \sigma_{3}^{2}\right] d t-\left[\sigma_{1}(a+b) d B_{1}\right. \\
& \left.+\sigma_{2} d B_{2}+c \sigma_{3} d B_{3}\right]=\left[-\beta S-\frac{a \mu}{S}-\frac{b \mu}{S}-\eta \beta V\right. \\
& +\frac{c \phi S}{V}+\left(\mu+\gamma+\frac{\sigma_{2}^{2}}{2}\right)+\beta(a+b+\eta c) I \\
& +a\left(\mu+\phi+\frac{\sigma_{1}^{2}}{2}\right)+b\left(\mu+\phi+\frac{\sigma_{1}^{2}}{2}\right) \\
& \left.+c \sigma_{3} d B_{3}\right] \\
& \left.+c\left(\mu+\frac{\sigma_{3}^{2}}{2}\right)\right] d t-\left[\sigma_{1}(a+b) d B_{1}+\sigma_{2} d B_{2}\right. \\
& \left.+c \sigma_{3} d B_{3}\right] \leq[-2 \sqrt{a \beta \mu}-3 \sqrt[3]{b c \eta \beta \mu \phi} \\
& +
\end{aligned}
$$

Let

$$
\begin{aligned}
& a=\frac{\beta \mu}{\left(\mu+\phi+\sigma_{1}^{2} / 2\right)^{2}}, \\
& b=\frac{\eta \beta \mu \phi}{\left(\mu+\phi+\sigma_{1}^{2} / 2\right)^{2}\left(\mu+\sigma_{3}^{2} / 2\right)}, \\
& c=\frac{\eta \beta \mu \phi}{\left(\mu+\phi+\sigma_{1}^{2} / 2\right)\left(\mu+\sigma_{3}^{2} / 2\right)^{2}} .
\end{aligned}
$$

Then

$$
\begin{aligned}
& d V_{1} \leq\left[-\frac{\beta \mu}{\mu+\phi+\sigma_{1}^{2} / 2}-\frac{\eta \beta \mu \phi}{\left(\mu+\phi+\sigma_{1}^{2} / 2\right)\left(\mu+\sigma_{3}^{2} / 2\right)}\right. \\
& \left.+\left(\mu+\gamma+\frac{\sigma_{2}^{2}}{2}\right)+\beta(a+b+\eta c) I\right] d t \\
& \quad-\left[\sigma_{1}(a+b) d B_{1}+\sigma_{2} d B_{2}+c \sigma_{3} d B_{3}\right]
\end{aligned}
$$

By integrating the last inequality, we obtain

$$
\begin{aligned}
& \frac{V_{1}(S(t), I(t), V(t))-V_{1}(S(0), I(0), V(0))}{t} \\
& \leq-\left(\mu+\gamma+\frac{\sigma_{2}^{2}}{2}\right)\left(R_{0}^{S}-1\right)+\beta(a+b+\eta c)\langle I\rangle \\
& \quad-\frac{1}{t} \int_{0}^{t} \sigma_{1}(a+b) d B_{1}(s)+\sigma_{2} d B_{2}(s)_{2} \\
& \quad+c \sigma_{3} d B_{3}(s) .
\end{aligned}
$$

By applying Theorem 1 and the strong law of large numbers for martingales [22], we get the desired result.

\section{Stationary Distribution}

In this section, by using the theory of Has'minski [18], we prove the existence of a unique ergodic stationary distribution, which indicates that the disease is persistent.

Theorem 6. If $R_{0}^{S}>1$, then system (3) has a unique stationary distribution and it has the ergodic property.

Proof. To prove Theorem 6, it is sufficient to verify assumptions (H.1) and (H.2).

(i) To verify (H.2), we show that there exists a neighborhood $D \subset \mathbb{R}_{+}^{3}$ and a nonnegative $\mathscr{C}^{2}$-function $V$ such that $L V(X)$ is negative for any $X \in \mathbb{R}_{+}^{3} \backslash D$. To this end, we define a $\mathscr{C}^{2}$-function in the form

$$
\begin{aligned}
W(S, I, V)= & M V_{1}(S, I, V)+V_{2}(S, I, V)-\log S \\
& -\log V,
\end{aligned}
$$

where $V_{1}$ is the same function defined in Section $3, V_{2}=$ : $(1 /(1+\theta))(S+I+V)^{1+\theta}$, and $M, \theta$ are positive constants that satisfy

$$
\begin{aligned}
- & M\left(\mu+\gamma+\frac{\sigma_{2}^{2}}{2}\right)\left(R_{0}^{S}-1\right)+A+2 \mu+\phi+\frac{\sigma_{1}^{2}}{2}+\frac{\sigma_{3}^{2}}{2} \\
& <0, \\
\mu & >\frac{\theta}{2} \max \left(\sigma_{1}^{2}, \sigma_{2}^{2}, \sigma_{3}^{2}\right),
\end{aligned}
$$

where

$$
A=\sup _{(S, I, V) \in \mathbb{R}_{+}^{3}}\left\{\mu(S+I+V)^{\theta}-\frac{\rho}{2}(S+I+V)^{\theta+1}\right\} .
$$


It is easy to see that

$$
\begin{aligned}
& \lim _{\left(S^{2}+I^{2}+V^{2}\right) \longrightarrow \infty} W(S, I, V)=\lim _{\left(S^{2}+I^{2}+V^{2}\right) \longrightarrow 0} W(S, I, V) \\
& =+\infty
\end{aligned}
$$

Moreover, $W(S, I, V)$ is a continuous function. Hence, $W(S, I, V)$ must have a minimum point $(\bar{S}, \bar{I}, \bar{V})$ in the interior of $\mathbb{R}_{+}^{3}$. Then we define a nonnegative $\mathscr{C}^{2}$-function $V$ as

$$
V(S, I, V)=W(S, I, V)-W(\bar{S}, \bar{I}, \bar{V}) .
$$

From the proof of Theorem 5, we have

$$
\begin{aligned}
L V_{1}(S, I, V) \leq & -\left(\mu+\gamma+\frac{\sigma_{2}^{2}}{2}\right)\left(R_{0}^{S}-1\right) \\
& +\beta(a+b+\eta c) I
\end{aligned}
$$

The operator $L$ defined in Section 2 acts on $V_{2},-\log S$, and $-\log V$ as follows:

$$
\begin{aligned}
L( & -\log S)=-\frac{\mu}{S}+\mu+\phi+\beta I+\frac{\sigma_{1}^{2}}{2}, \\
L(-\log V)=-\frac{\phi S}{V}+\mu+\eta \beta I+\frac{\sigma_{3}^{2}}{2} & \\
L V_{2}(S, I, V) & (S+I+V)^{\theta}[\mu-\mu(S+I+V)-\gamma I] \\
= & \frac{\theta}{2}(S+I+V)^{\theta-1}\left(\sigma_{1}^{2} S^{2}+\sigma_{2}^{2} I^{2}+\sigma_{3}^{2} V^{2}\right) \\
\leq & \mu(S+I+V)^{\theta}-\mu(S+I+V)^{\theta+1} \\
& +\frac{\theta}{2}\left(\sigma_{1}^{2} \vee \sigma_{2}^{2} \vee \sigma_{3}^{2}\right)(S+I+V)^{\theta+1} \\
= & \mu(S+I+V)^{\theta} \\
& -\left[\mu-\frac{\theta}{2}\left(\sigma_{1}^{2} \vee \sigma_{2}^{2} \vee \sigma_{3}^{2}\right)\right](S+I+V)^{\theta+1} \\
= & \mu(S+I+V)^{\theta}-\rho(S+I+V)^{\theta+1} \\
\leq & A-\frac{\rho}{2}\left(S^{1+\theta}+I^{1+\theta}+V^{1+\theta}\right),
\end{aligned}
$$

where

$$
\rho=\mu-\frac{\theta}{2}\left(\sigma_{1}^{2} \vee \sigma_{2}^{2} \vee \sigma_{3}^{2}\right)
$$

Therefore

$$
\begin{aligned}
L V(S, I, V) \leq & f(S)+g(I)-\frac{\rho}{2} V^{1+\theta}-\frac{\phi S}{V} \\
& -M\left(\mu+\gamma+\frac{\sigma_{2}^{2}}{2}\right)\left(R_{0}^{S}-1\right)+A+2 \mu \\
& +\phi+\frac{\sigma_{1}^{2}}{2}+\frac{\sigma_{3}^{2}}{2}
\end{aligned}
$$

where

$$
\begin{aligned}
& f(S)=-\frac{\mu}{S}-\frac{\rho}{2} S^{1+\theta} \\
& g(I)=\beta[M(a+b+\eta c)+1+\eta] I-\frac{\rho}{2} I^{1+\theta} .
\end{aligned}
$$

Next, we construct the following compact subset:

$$
D=\left\{\epsilon \leq S \leq \frac{1}{\epsilon}, \epsilon^{2} \leq V \leq \frac{1}{\epsilon^{2}}, \epsilon \leq I \leq \frac{1}{\epsilon}\right\}
$$

where $\epsilon$ is a sufficiently small positive number, satisfying the following inequalities:

$$
\begin{array}{r}
\stackrel{\vee}{g}-\frac{\mu}{\epsilon}<0, \\
\vee \\
f+\beta[M(a+b+\eta c)+1+\eta] \epsilon<0, \\
\vee \\
f+\stackrel{\vee}{ }-\frac{\phi}{\epsilon}<0, \\
\stackrel{\vee}{g}-\frac{\rho}{2} \frac{1}{\epsilon^{1+\theta}}<0, \\
\vee \\
f+B-\frac{\rho}{4} \frac{1}{\epsilon^{1+\theta}}<0, \\
\vee \\
f+\stackrel{\rho}{g}-\frac{\rho}{2} \frac{1}{\epsilon^{2(1+\theta)}}<0,
\end{array}
$$

where the constant $B$ will be determined later.

Then

$$
\mathbb{R}_{+}^{3} \backslash D=D_{1}^{c} \cup D_{2}^{c} \cup D_{3}^{c} \cup D_{4}^{c} \cup D_{5}^{c} \cup D_{6}^{c},
$$

with

$$
\begin{aligned}
& D_{1}^{c}=\left\{(S, I, V) \in \frac{\mathbb{R}_{+}^{3}}{0}<S<\epsilon\right\}, \\
& D_{2}^{c}=\left\{(S, I, V) \in \frac{\mathbb{R}_{+}^{3}}{0}<I<\epsilon\right\}, \\
& D_{3}^{c}=\left\{(S, I, V) \in \frac{\mathbb{R}_{+}^{3}}{0}<V<\epsilon^{2}, S>\epsilon\right\}, \\
& D_{4}^{c}=\left\{(S, I, V) \in \frac{\mathbb{R}_{+}^{3}}{S}>\frac{1}{\epsilon}\right\}, \\
& D_{5}^{c}=\left\{(S, I, V) \in \frac{\mathbb{R}_{+}^{3}}{I}>\frac{1}{\epsilon}\right\}, \\
& D_{6}^{c}=\left\{(S, I, V) \in \frac{\mathbb{R}_{+}^{3}}{V}>\frac{1}{\epsilon^{2}}\right\} .
\end{aligned}
$$


Now, we will show that $L V$ is negative for any $(S, I, V) \in \mathbb{R}_{+}^{3} \backslash$ D.

Case 1. If $(S, I, V) \in D_{1}^{c}$, we obtain from (36) that

$$
\begin{aligned}
L V(S, I, V) \leq & -M\left(\mu+\gamma+\frac{\sigma_{2}^{2}}{2}\right)\left(R_{0}^{S}-1\right)+A+2 \mu \\
& +\phi+\frac{\sigma_{1}^{2}}{2}+\frac{\sigma_{3}^{2}}{2}+\stackrel{\vee}{g}-\frac{\mu}{\epsilon}<0 .
\end{aligned}
$$

Case 2. If $(S, I, V) \in D_{2}^{c}$, (37) implies that

$$
\begin{aligned}
L V(S, I, V) \leq & -M\left(\mu+\gamma+\frac{\sigma_{2}^{2}}{2}\right)\left(R_{0}^{S}-1\right)+A+2 \mu \\
& +\phi+\frac{\sigma_{1}^{2}}{2}+\frac{\sigma_{3}^{2}}{2}+\stackrel{\vee}{f} \\
& +\beta[M(a+b+\eta c)+1+\eta] \epsilon<0 .
\end{aligned}
$$

Case 3. If $(S, I, V) \in D_{3}^{c}$, from (38) it follows that

$$
\begin{aligned}
L V(S, I, V) \leq & -M\left(\mu+\gamma+\frac{\sigma_{2}^{2}}{2}\right)\left(R_{0}^{S}-1\right)+A+2 \mu \\
& +\phi+\frac{\sigma_{1}^{2}}{2}+\frac{\sigma_{3}^{2}}{2}+\stackrel{\vee}{f}+\stackrel{\vee}{g}-\frac{\phi}{\epsilon}<0 .
\end{aligned}
$$

Case 4. If $(S, I, V) \in D_{4}^{c}$, (39) implies that

$$
\begin{aligned}
L V(S, I, V) \leq & -M\left(\mu+\gamma+\frac{\sigma_{2}^{2}}{2}\right)\left(R_{0}^{S}-1\right)+A+2 \mu \\
& +\phi+\frac{\sigma_{1}^{2}}{2}+\frac{\sigma_{3}^{2}}{2}+\stackrel{\vee}{g}-\frac{\rho}{2} \frac{1}{\epsilon^{1+\theta}}<0 .
\end{aligned}
$$

Case 5. If $(S, I, V) \in D_{5}^{c}$, from (40) we get

$$
\begin{aligned}
L V(S, I, V) \leq & -M\left(\mu+\gamma+\frac{\sigma_{2}^{2}}{2}\right)\left(R_{0}^{S}-1\right)+A+2 \mu \\
& +\phi+\frac{\sigma_{1}^{2}}{2}+\frac{\sigma_{3}^{2}}{2}+\stackrel{\vee}{f}+B-\frac{\rho}{4} \frac{1}{\epsilon^{1+\theta}}<0,
\end{aligned}
$$

where

$$
B=\sup _{I \in(0, \infty)}\left\{\beta[M(a+b+\eta c)+1+\eta] I-\frac{\rho}{4} I^{1+\theta}\right\} .
$$

Case 6. If $(S, I, V) \in D_{6}^{c}$, from (41) we obtain

$$
\begin{aligned}
L V(S, I, V) \leq & -M\left(\mu+\gamma+\frac{\sigma_{2}^{2}}{2}\right)\left(R_{0}^{S}-1\right)+A+2 \mu \\
& +\phi+\frac{\sigma_{1}^{2}}{2}+\frac{\sigma_{3}^{2}}{2}+\stackrel{\vee}{f}+\stackrel{\vee}{g}-\frac{\rho}{2} \frac{1}{\epsilon^{2(1+\theta)}}
\end{aligned}
$$

From the previous discussion, we have

$$
L V(S, I, V)<0, \quad(S, I, V) \in \mathbb{R}_{+}^{3} \backslash D
$$

(ii) Now, we verify assumption (H.1). The diffusion matrix of system (3) is

$$
\tilde{A}=\left(\begin{array}{ccc}
\sigma_{1}^{2} S^{2} & 0 & 0 \\
0 & \sigma_{2}^{2} I^{2} & 0 \\
0 & 0 & \sigma_{3}^{2} V^{2}
\end{array}\right)
$$

There is $\lambda=\min \left(\sigma_{1}^{2} S^{2}, \sigma_{2}^{2} I^{2}, \sigma_{3}^{2} V^{2}\right)>0$ such that

$$
\begin{aligned}
\sum_{i, j=1}^{3} \tilde{a}_{i j}(X) \xi_{i} \xi_{j} & =\sigma_{1}^{2} S^{2} \xi_{1}^{2}+\sigma_{2}^{2} S I^{2} \xi_{2}^{2}+\sigma_{3}^{2} V^{2} \xi_{3}^{2} \\
& \geq \lambda|\xi|^{2}
\end{aligned}
$$

for $(S, I, V) \in \bar{D}$ and $\xi \in \mathbb{R}_{+}^{3}$. That is to say, assumption (H.1) holds.

Consequently, system (3) has an ergodic stationary distribution.

\section{Extinction}

Theorem 7. We assume that $\mu>\max \left(\sigma_{1}^{2}, \sigma_{2}^{2}, \sigma_{3}^{2}\right) / 2$. Let $(S(t), I(t), V(t)) \in \mathbb{R}_{+}^{3}$ be the solution of system (3). If $\tilde{R}_{0}^{S}<1$, then the disease dies out with probability one.

The threshold $R_{0}^{S}$ is defined as follows:

$$
\begin{aligned}
\tilde{R_{0}^{S}}= & \frac{\beta \mu}{(\mu+\phi)\left(\mu+\gamma+\sigma_{2}^{2} / 2\right)} \\
& +\frac{\eta \beta \phi}{(\mu+\phi)\left(\mu+\gamma+\sigma_{2}^{2} / 2\right)}
\end{aligned}
$$

Proof. By integrating system (3), we obtain

$$
\begin{aligned}
& \frac{S(t)-S(0)}{t}+\frac{I(t)-I(0)}{t}+\frac{V(t)-V(0)}{t}=\mu \\
& -\mu\langle S\rangle-(\mu+\gamma)\langle I\rangle-\mu\langle V\rangle \\
& \quad+\frac{1}{t}\left[\sigma_{1} \int_{0}^{t} S(r) d B_{1}(r)+\sigma_{2} \int_{0}^{t} I(r) d B_{2}(r)\right. \\
& \left.+\sigma_{3} \int_{0}^{t} v(r) d B_{3}(r)\right] .
\end{aligned}
$$


It follows that

$$
\langle V\rangle=1-\langle S\rangle-\frac{\mu+\gamma}{\mu}\langle I\rangle+\psi_{1}(t)
$$

where

$$
\begin{aligned}
& \psi_{1}(t)=-\left(\frac{S(t)-S(0)}{t}+\frac{I(t)-I(0)}{t}\right. \\
& \left.+\frac{V(t)-V(0)}{t}\right)+\frac{1}{\mu t}\left[\sigma_{1} \int_{0}^{t} S(r) d B_{1}(r)\right. \\
& \left.+\sigma_{2} \int_{0}^{t} I(r) d B_{2}(r)+\sigma_{3} \int_{0}^{t} v(r) d B_{3}(r)\right] .
\end{aligned}
$$

From the first equation of system (3), we obtain

$$
\langle S\rangle=\frac{\mu}{\mu+\phi}-\frac{\beta}{\mu+\phi}\langle S I\rangle+\psi_{2}(t),
$$

where

$$
\psi_{2}(t)=-\frac{S(t)-S(0)}{(\mu+\phi) t}+\frac{\sigma_{1}}{(\mu+\phi) t} \int_{0}^{t} S(s) d B_{1}(s) .
$$

Applying Itô's formula to system (3), one obtains

$$
\begin{aligned}
\frac{\log I(t)-\log I(0)}{t}= & \beta\langle S\rangle+\eta \beta\langle V\rangle-(\mu+\gamma)-\frac{\sigma_{2}^{2}}{2} \\
& +\frac{\sigma_{2} B_{2}(t)}{t} .
\end{aligned}
$$

By injecting (56) and (58) into (60), we have

$$
\begin{aligned}
\frac{\log I(t)-\log I(0)}{t}= & \beta \frac{\mu+\eta \phi}{\mu+\phi}-\left(\mu+\gamma+\frac{\sigma_{2}^{2}}{2}\right) \\
& +\beta(1-\eta) \psi_{1}(t)+\eta \beta \psi_{2}(t) \\
& -\beta^{2} \frac{1-\eta}{\mu+\phi}\langle S I\rangle \\
& -\beta \frac{\eta(\mu+\gamma)}{\mu}\langle I\rangle+\frac{\sigma_{2} B_{2}(t)}{t} \\
\leq & \beta \frac{\mu+\eta \phi}{\mu+\phi}-\left(\mu+\gamma+\frac{\sigma_{2}^{2}}{2}\right) \\
& +\beta(1-\eta) \psi_{1}(t)+\eta \beta \psi_{2}(t) \\
& +\frac{\sigma_{2} B_{2}(t)}{t} .
\end{aligned}
$$

From the strong law of large numbers for martingales [22], we get

$$
\lim _{t \rightarrow \infty} \frac{B_{2}(t)}{t}=0, \quad \text { a.s. }
$$

By Theorem 1, we get

$$
\lim _{t \longrightarrow \infty} \psi_{1}(t)=\lim _{t \longrightarrow \infty} \psi_{2}(t)=0
$$

By taking the superior limit of both sides of (61), we obtain

$$
\begin{aligned}
\limsup _{t \rightarrow \infty} \frac{\log I(t)}{t} & \leq \frac{\beta \mu}{\mu+\phi}+\frac{\eta \beta \phi}{\mu+\phi}-\left(\mu+\gamma+\frac{\sigma_{2}^{2}}{2}\right) \\
& =\left(\mu+\gamma+\frac{\sigma_{2}^{2}}{2}\right)\left(\widetilde{R_{0}^{S}}-1\right) .
\end{aligned}
$$

This finishes the proof.

Theorem 8. We assume that $\sigma_{1}=\sigma_{3}=\sigma$ and $B_{1}(t)=B_{3}(t)=$ $B(t)$. Let $(S(t), I(t), V(t)) \in \mathbb{R}_{+}^{3}$ be the solution of system (3). If $\sigma^{2}>2 \eta \phi$, then

$$
\begin{aligned}
& \limsup _{t \rightarrow \infty} \frac{\log I(t)}{t} \\
& \quad \leq(\mu+\gamma)\left(\frac{\beta}{\mu+\gamma} \int_{0}^{\infty} x \pi(x) d x-1\right) \text { a.s. }
\end{aligned}
$$

If $(\beta /(\mu+\gamma)) \int_{0}^{\infty} x \pi(x) d x<1$, then

$$
\lim _{t \rightarrow \infty} I(t)=0 \quad \text { a.s. }
$$

and the distribution of $S(t)+\eta V(t)$ converges weakly to the measure that has the density given by

$$
\pi(x)=\frac{Q}{\sigma^{2}} x^{-2\left(1-\eta \phi / \sigma^{2}\right)} e^{-2 \mu / \sigma^{2} x}, \quad x \in(0 . \infty),
$$

where

$$
Q=\sigma^{2}\left(\frac{2 \mu}{\sigma^{2}}\right)^{1-2\left(\eta \phi / \sigma^{2}\right)} \Gamma^{-1}\left(1-2 \frac{\eta \phi}{\sigma^{2}}\right) .
$$

Proof. We assume that $\sigma_{1}=\sigma_{3}=\sigma$ and $B_{1}(t)=B_{3}(t)=B(t)$. From system (3), we have

$$
\begin{aligned}
d(S(t)+\eta V(t)) \leq & {[\mu+\eta \phi(S(t)+\eta V(t))] d t } \\
& +\sigma_{1} S(t) d B_{1}(t) \\
& +\eta V(t) \sigma_{3} d B_{3}(t), \\
= & d X(t),
\end{aligned}
$$

where

$$
d X(t)=[\mu+\eta \phi X(t)] d t+\sigma X(t) d B(t),
$$

with the initial value $X(0)=S(0)+\eta V(0)$.

We compute that

$$
\begin{aligned}
\int \frac{\mu+\eta \phi x}{\sigma^{2} x^{2}} d x & =\frac{1}{\sigma^{2}} \int\left(\frac{\mu}{x^{2}}+\frac{\eta \phi}{x}\right) d x \\
& =\frac{1}{\sigma^{2}}\left[-\frac{\mu}{x}+\eta \phi \ln x\right]+B,
\end{aligned}
$$

where $\mathrm{B}$ is a constant. 
One can see that

$$
\begin{aligned}
\int_{0}^{\infty} & x^{-2\left(1-\eta \phi / \sigma^{2}\right)} e^{-2 \mu / \sigma^{2} x} d x \\
& =\left(\frac{2 \mu}{\sigma^{2}}\right)^{2\left(\eta \phi / \sigma^{2}\right)-1} \int_{0}^{\infty} y^{\left(1-2\left(\eta \phi / \sigma^{2}\right)\right)-1} e^{-y} d y \\
& =\left(\frac{2 \mu}{\sigma^{2}}\right)^{2\left(\eta \phi / \sigma^{2}\right)-1} \Gamma\left(1-2 \frac{\eta \phi}{\sigma^{2}}\right),
\end{aligned}
$$

where $\Gamma$ is the Gamma function defined by

$$
\Gamma(Z)=\int_{0}^{\infty} x^{z-1} e^{-x} d x
$$

If $\sigma^{2}>2 \eta \phi$, then

$$
\int_{0}^{\infty} x^{-2\left(1-\eta \phi / \sigma^{2}\right)} e^{-2 \mu / \sigma^{2} x} d x<\infty
$$

Thus, condition (H.3) in Lemma 3 is verified. So, system (70) has the ergodic property and the invariant density given by

$$
\pi(x)=\frac{Q}{\sigma^{2}} x^{-2\left(1-\eta \phi / \sigma^{2}\right)} e^{-2 \mu / \sigma^{2} x}, \quad x \in(0, \infty) .
$$

Let us compute $Q$.

According to

$$
\int_{0}^{\infty} \pi(x) d x=1
$$

one has

$$
Q=\sigma^{2}\left(\frac{2 \mu}{\sigma^{2}}\right)^{1-2\left(\eta \phi / \sigma^{2}\right)} \Gamma^{-1}\left(1-2 \frac{\eta \phi}{\sigma^{2}}\right) .
$$

From the ergodic theorem, it follows that

$$
\lim _{t \rightarrow \infty} \frac{1}{t} \int_{0}^{t} X(r) d r=\int_{0}^{\infty} x \pi(x) d x \quad \text { a.s. }
$$

On the other hand, by applying Itô's formula to $\log I(t)$ and then integrating, one has

$$
\begin{aligned}
& \frac{\log I(t)-\log I(0)}{t} \\
& =\frac{\beta}{t} \int_{0}^{t}(S(r)+\eta V(r)) d r-(\mu+\gamma) \\
& \quad-\frac{\sigma^{2}}{2} \int_{0}^{t}(S(r)+\eta V(r))^{2} d r+\frac{1}{t} M(t),
\end{aligned}
$$

where $M(t)=\sigma \int_{0}^{t}(S(r)+\eta V(r)) d B(r)$ whose quadratic variation is

$$
\langle M, M\rangle(t)=\sigma^{2} \int_{0}^{t}(S(r)+\eta V(r))^{2} d r .
$$

In view of the exponential martingales inequality [22], for any positive constants $T, \alpha$, and $\nu$, we have

$$
\mathbb{P}\left\{\sup _{0 \leq t \leq T}\left[M(t)-\frac{\alpha}{2}\langle M, M\rangle(t)\right]>v\right\} \leq e^{-\alpha \nu} .
$$

Choosing $T=k, \alpha=1$ and $v=2 \log k$, one has

$$
\mathbb{P}\left\{\sup _{0 \leq t \leq k}\left[M(t)-\frac{1}{2}\langle M, M\rangle(t)\right]>2 \log k\right\} \leq \frac{1}{k^{2}} .
$$

Applying the Borel-Cantelli Lemma [22] leads to the fact that, for almost all $\omega \in \Omega$, there exists a random integer $k_{0}=k_{0}(\omega)$ such that, for any $k \geq k_{0}$, we obtain

$$
\sup _{0 \leq t \leq k}\left[M(t)-\frac{1}{2}\langle M, M\rangle(t)\right] \leq 2 \log k .
$$

That is,

$$
M(t) \leq \frac{1}{2}\langle M, M\rangle(t)+2 \log k \quad \text { for all } t \leq k .
$$

Substituting this inequality into (79) yields

$$
\begin{aligned}
& \frac{\log I(t)-\log I(0)}{t} \\
& \leq \frac{\beta}{t} \int_{0}^{t}(S(r)+\eta V(r)) d r-(\mu+\gamma)+2 \frac{\log k}{t}
\end{aligned}
$$

for all $t \leq k$.

Applying the comparison theorem of stochastic differential equations on inequality (69) gives

$$
S(t)+\eta V(t) \leq X(t) \quad \text { a.s. }
$$

It follows that

$$
\begin{aligned}
\frac{\log I(t)-\log I(0)}{t} \leq & \frac{\beta}{t} \int_{0}^{t} X(r) d r-(\mu+\gamma) \\
& +2 \frac{\log k}{t}, \quad \text { for all } t \leq k
\end{aligned}
$$

Taking the superior limit on both sides of the previous inequality leads to

$$
\begin{aligned}
& \limsup _{t \rightarrow \infty} \frac{\log I(t)-\log I(0)}{t} \\
& \quad \leq \beta \int_{0}^{\infty} x \pi(x) d x-(\mu+\gamma) \text { a.s. }
\end{aligned}
$$

If $(\beta /(\mu+\gamma)) \int_{0}^{\infty} x \pi(x) d x<1$, we conclude that

$$
\lim _{t \rightarrow \infty} I(t)=0 \quad \text { a.s. }
$$

As a result, for any small $\epsilon>0$, there exist $t_{0}$ and a set $\Omega_{\epsilon} \subset \Omega$ such that $\mathbb{P}\left(\Omega_{\epsilon}\right)>1-\epsilon$ and $\beta\left(S+\eta^{2} V\right) I \leq \epsilon\left(S+\eta^{2} V\right) \leq$ $\epsilon(S+\eta V)$ for all $t \geq t_{0}$ and $\omega \in \Omega_{\epsilon}$. From

$$
\begin{aligned}
& {[\mu-\mu(S(t)+\eta V(t))-\epsilon(S(t)+\eta V(t))] d t} \\
& \quad+\sigma(S(t)+\eta V(t)) d B(t) \leq d(S(t)+\eta V(t)) \\
& \quad \leq[\mu-\mu(S(t)+\eta V(t))] d t \\
& \quad+\sigma(S(t)+\eta V(t)) d B(t),
\end{aligned}
$$

it follows that the distribution of the process $S(t)+\eta V(t)$ converges weakly to the measure that has the density $\pi$.

This completes the proof. 


\section{Numerical Simulations}

In this section, we present the numerical simulations to support the above analytical results, illustrating persistence in mean and extinction of the disease.

In the two following examples, we choose the initial value as $(S(0), I(0), V(0))=(0.2,0.2,0.2)$.

Example 9. In model (3), we choose the parameters as follows:

$$
\begin{gathered}
\sigma_{1}=0.2, \\
\sigma_{2}=0.2, \\
\sigma_{3}=0.2, \\
\beta=10, \\
\mu=1, \\
\phi=15, \\
\gamma=4, \\
\eta=0.6 .
\end{gathered}
$$

We compute that

$$
R_{0}^{S}=1.2215>1 .
$$

Then, according to Theorem 5, the disease is persistent (see Figure 1). Furthermore, by Theorem 6, system (3) has a unique stationary distribution (see Figure 2).

Example 10. We choose the parameters as follows:

$$
\begin{gathered}
\sigma_{1}=0.6, \\
\sigma_{2}=1, \\
\sigma_{3}=0.8, \\
\beta=8, \\
\mu=1, \\
\phi=15, \\
\gamma=4, \\
\eta=0.6 .
\end{gathered}
$$

Then

$$
\tilde{R_{0}^{S}}=0.9091<1
$$

Theorem 7 implies that system (3) has disease extinction (see Figure 3).
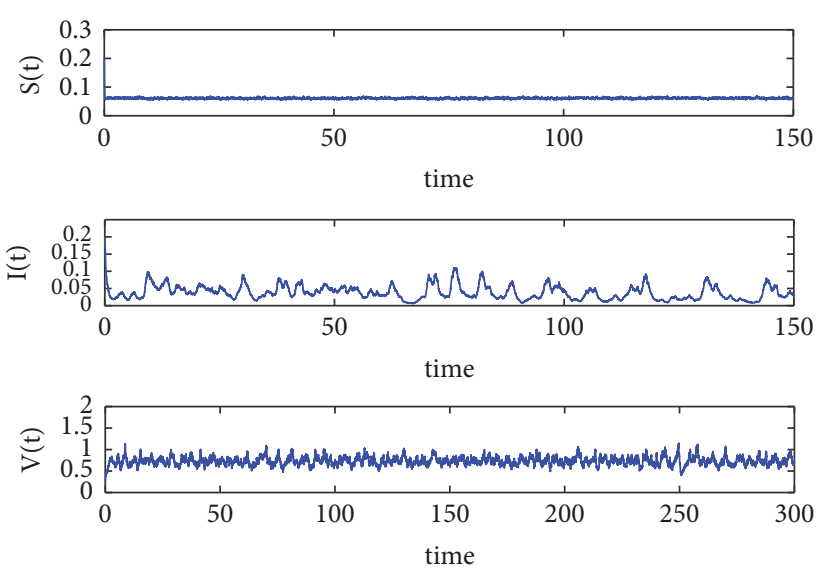

FIgURE 1: Simulation of the paths $S(t), I(t)$, and $V(t)$ for model (3).

Example 11. We choose the parameters as follows:

$$
\begin{aligned}
\sigma_{1} & =\sigma_{3}=\sigma=1.15, \\
\beta & =10, \\
\mu & =1, \\
\phi & =1, \\
\gamma & =4, \\
\eta & =0.2 .
\end{aligned}
$$

Then

$$
\sigma^{2}>2 \eta \phi
$$

$$
\frac{\beta}{\mu+\gamma} \int_{0}^{\infty} x \pi(x) d x<1
$$

According to Theorem 8, we get

$$
\lim _{t \rightarrow \infty} I(t)=0 \quad \text { a.s. }
$$

It follows that the distribution of the process $S(t)+\eta V(t)$ converges weakly to the measure that has the density $\pi$ (see Figure 4).

\section{Conclusion}

This paper is concerned with the dynamics of a stochastic SIVR epidemic model with multiplicative noise sources. By constructing a convenient positive function, we establish sufficient conditions for the existence of a unique ergodic stationary distribution to model (3) and we prove that the disease will be permanent. In addition, we also establish sufficient conditions for extinction of the disease.

Many works have been done to study the continuous stochastic models by using the white noise. Sometimes, population systems may suffer sudden environmental perturbations, such as SARS, floods, and toxic pollutants. These phenomena cannot be modeled by stochastic continuous 

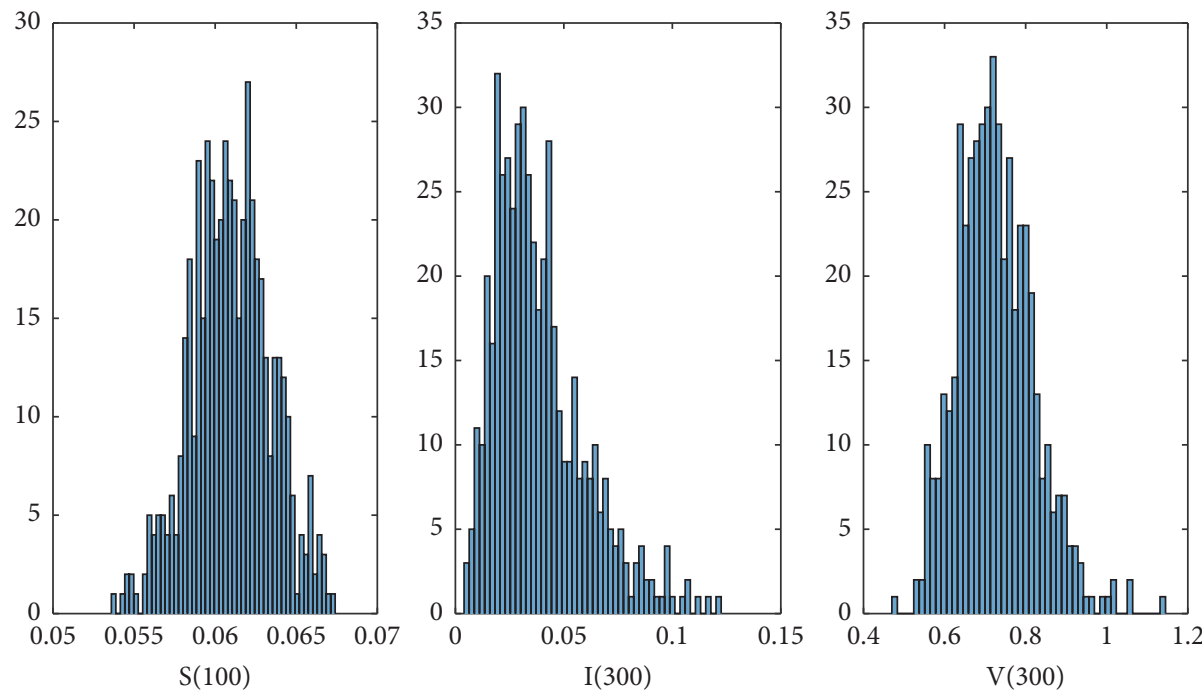

Figure 2: Distribution of $S(300), I(300)$, and $V(300)$.
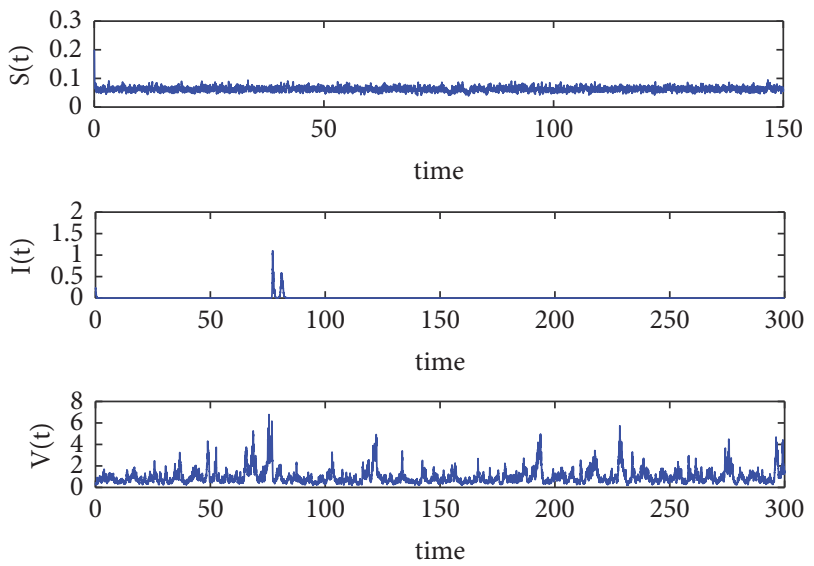

FIgURE 3: Simulation of the paths $S(t), I(t)$, and $V(t)$ for model (3).

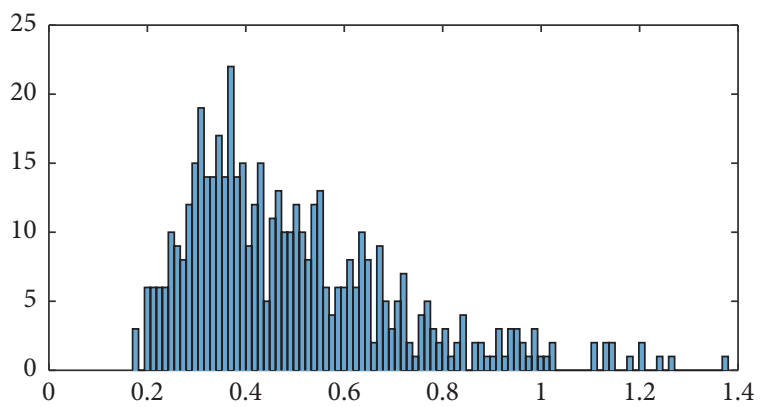

FIGURE 4: Distribution of $S(t)+\eta V(t)$ at time $t=10000$.

models. During the last years, there was an increasing interest in Lévy noise that has non-Gaussian statistics. A lot of works have been realized and have shown the effectiveness of the Lévy noise in studying such phenomena (see [25-27]). We investigate in our future works the impact of the Lévy noise on the dynamic of complicated population systems.

\section{Data Availability}

No data were used to support this study.

\section{Conflicts of Interest}

The authors declare that there are no conflicts of interest regarding the publication of this paper.

\section{References}

[1] R. M. Anderson and R. M. May, "Population biology of infectious disease: Part I," Nature, vol. 280, pp. 361-376, 1979.

[2] R. M. May and R. M. Anderson, "Population biology of infectious disease: Part II," Nature, vol. 280, no. 5722, pp. 455$461,1979$.

[3] W. O. Kermack and A. G. McKendrick, "A contribution to the mathematical theory of epidemics," Proceedings of the Royal Society A Mathematical, Physical and Engineering Sciences, vol. 115 , no. 772, pp. 700-721, 1927.

[4] H. W. Hethcote, "The mathematics of infectious diseases," SIAM Review, vol. 42, no. 4, pp. 599-653, 2000.

[5] A. Korobeinikov, P. K. Maini, and W. J. Walker, "Estimation of effective vaccination rate: pertussis in New Zealand as a case study," Journal of Theoretical Biology, vol. 224, no. 2, pp. 269275, 2003.

[6] J. Li and Z. Ma, "Qualitative analyses of SIS epidemic model with vaccination and varying total population size," Mathematical and Computer Modelling, vol. 35, no. 11-12, pp. 1235-1243, 2002.

[7] C. Fraser, C. A. Donnelly, S. Cauchemez et al., "Pandemic potential of a strain of influenza A (H1N1): early findings," Science, vol. 324, no. 5934, pp. 1557-1561, 2009.

[8] J. Arino, K. L. Cooke, P. Van den Driessche, and J. VelascoHernández, "An epidemiology model that includes a leaky vaccine with a general waning function," Discrete and Continuous Dynamical Systems - Series B, vol. 4, no. 2, pp. 479-495, 2004.

[9] F. Brauer, "Backward bifurcations in simple vaccination models," Journal of Mathematical Analysis and Applications, vol. 298, no. 2, pp. 418-431, 2004. 
[10] Y. Cai, Y. Kang, M. Banerjee, and W. Wang, "A stochastic epidemic model incorporating media coverage," Communications in Mathematical Sciences, vol. 14, no. 4, pp. 893-910, 2016.

[11] T. C. Gard, "Persistence in stochastic food web models," Bulletin of Mathematical Biology, vol. 46, no. 3, pp. 357-370, 1984.

[12] C. Ji, D. Jiang, Q. Yang, and N. Shi, "Dynamics of a multigroup SIR epidemic model with stochastic perturbation," Automatica, vol. 48, no. 1, pp. 121-131, 2012.

[13] E. Renshaw, Modelling Biological Populations in Space and Time, vol. 11, Cambridge University Press, Cambridge, UK, 1991.

[14] D. Kiouach and Y. Sabbar, "Stability and threshold of a stochastic SIRS epidemic model with vertical transmission and transfer from infectious to susceptible individuals," Discrete Dynamics in Nature and Society, Article ID 7570296, 13 pages, 2018.

[15] O. A. Chichigina, A. A. Dubkov, D. Valenti, and B. Spagnolo, "Stability in a system subject to noise with regulated periodicity," Physical Review E: Statistical, Nonlinear, and Soft Matter Physics, vol. 84, no. 2, 2011.

[16] O. Chichigina, D. ValentI, and B. Spagnolo, "A simple noise model with memory for biological systems," Fluctuation and Noise Letters, vol. 52, pp. 243-250, 2005.

[17] B. Spagnolo, D. Valenti, and A. Fiasconaro, "Noise in ecosystems: a short review," Mathematical Biosciences and Engineering, vol. 1, no. 1, pp. 185-211, 2004.

[18] E. Tornatore, P. Vetro, and S. M. Buccellato, "SIVR epidemic model with stochastic perturbation," Neural Computing and Applications, vol. 24, no. 2, pp. 309-315, 2014.

[19] D. Zhao and S. Yuan, "Persistence and stability of the diseasefree equilibrium in a stochastic epidemic model with imperfect vaccine," Advances in Difference Equations, vol. 2016: Article ID 280, 14 pages, 2016.

[20] R. Rudnicki, "Long-time behaviour of a stochastic preypredator model," Stochastic Processes and Their Applications, vol. 108, no. 1, pp. 93-107, 2003.

[21] Y. Zhao and D. Jiang, "The threshold of a stochastic SIS epidemic model with vaccination," Applied Mathematics and Computation, vol. 243, pp. 718-727, 2014.

[22] X. Mao, Stochastic Differential Equations and Applications, Horwood, Chichester, UK, 2nd edition, 1997.

[23] R. Khasminskii, Stochastic Stability of Differential Equations, vol. 66, Springer, Berlin, Germany, 2nd edition, 2012.

[24] Y. A. Kutoyants, Statistical Inference for Ergodic Diffusion Processes, Springer, London, UK, 2004.

[25] A. La Cognata, D. Valenti, A. A. Dubkov, and B. Spagnolo, "Dynamics of two competing species in the presence of Lévy noise sources," Physical Review E: Statistical, Nonlinear, and Soft Matter Physics, vol. 82, no. 1, Article ID 011121, 2010.

[26] X. Zhang, D. Jiang, T. Hayat, and B. Ahmad, "Dynamics of a stochastic SIS model with double epidemic diseases driven by Lévy jumps," Physica A: Statistical Mechanics and its Applications, vol. 471, pp. 767-777, 2017.

[27] J. Yu and M. Liu, "Stationary distribution and ergodicity of a stochastic food-chain model with Lévy jumps," Physica A: Statistical Mechanics and its Applications, vol. 482, pp. 14-28, 2017. 


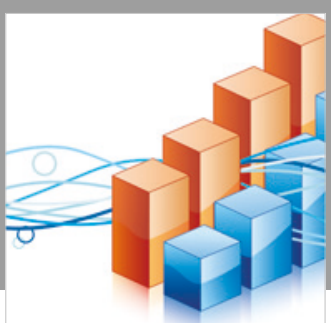

Advances in

Operations Research

\section{-n-m}
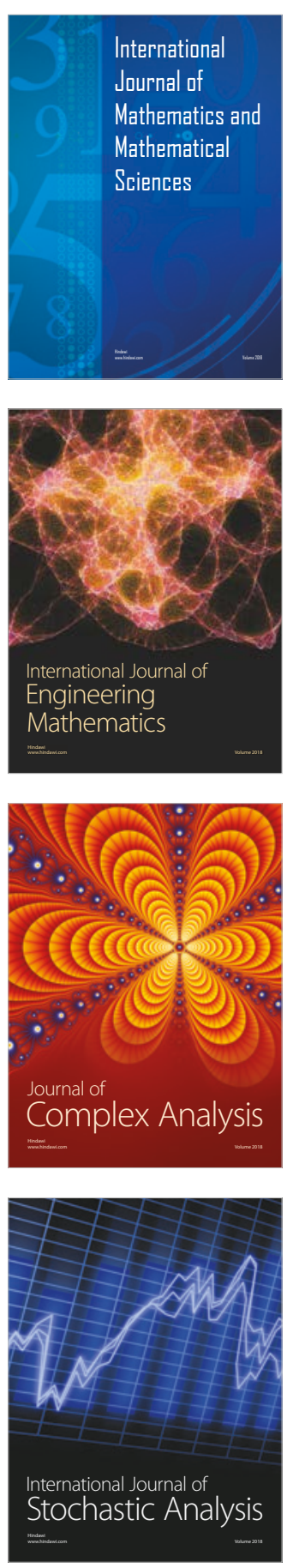
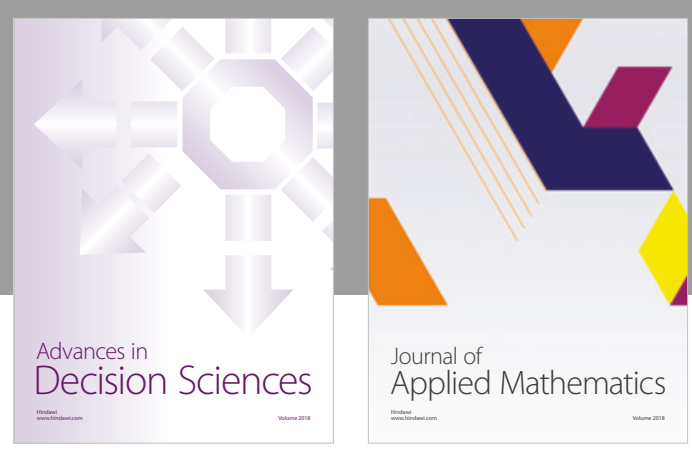

Journal of

Applied Mathematics
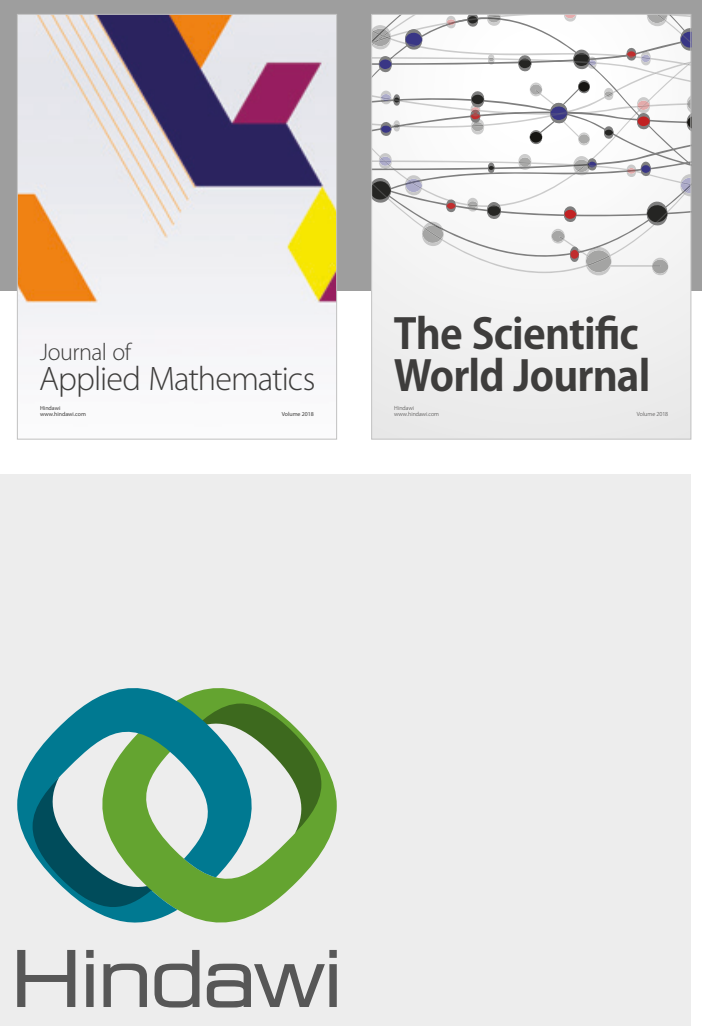

Submit your manuscripts at

www.hindawi.com

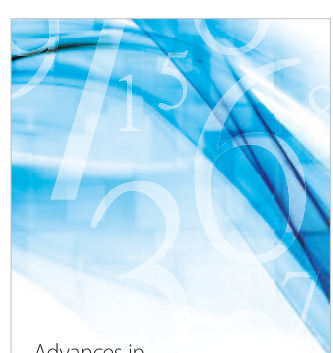

Advances in
Numerical Analysis
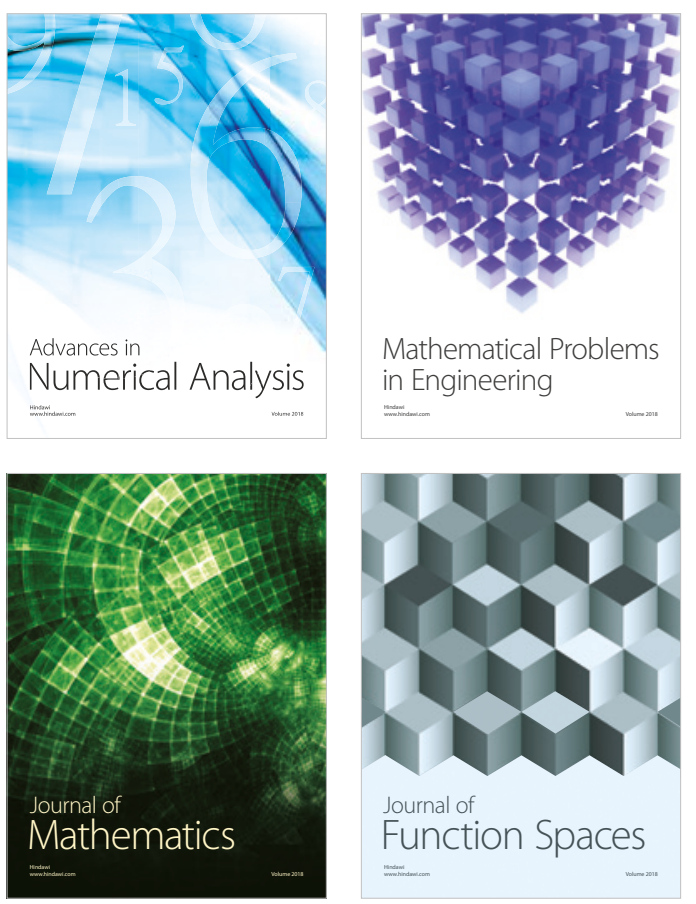

Mathematical Problems in Engineering

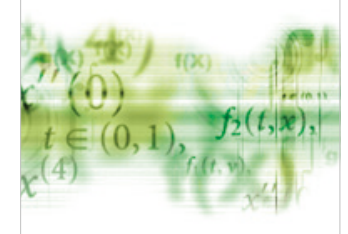

International Journal of

Differential Equations

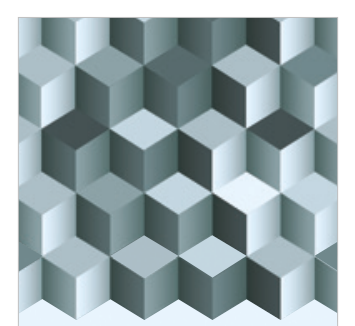

Journal of

Function Spaces

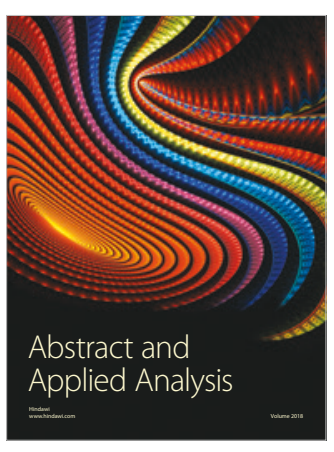

The Scientific

World Journal

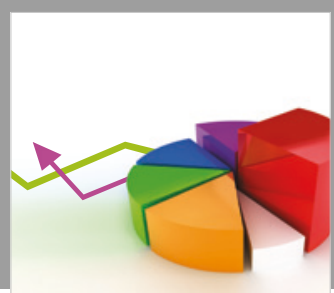

Journal of

Probability and Statistics
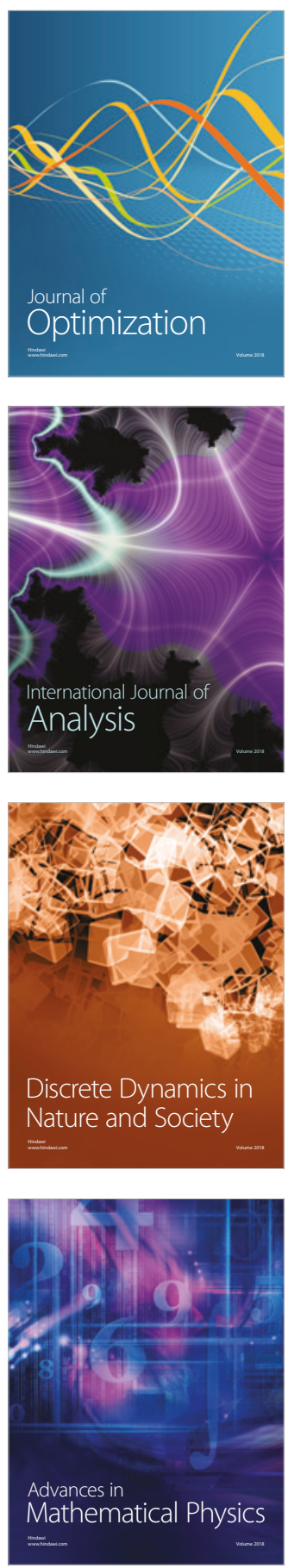